\title{
Utilización de Herramientas de Calidad para la Mejora en los Procesos de Extrusión de Plásticos
}

\section{Using Quality Tools to Improvement the Plastic Film Extrusion Processes}

\author{
DOI: $\underline{\text { http://dx.doi.org/10.17981/bilo }}$
}

Fecha de Recepción: 30/10/2019. Fecha de Aceptación: 11/12/2019. Fecha de Publicación: 16/12/2019

\author{
Alexander Troncoso-Palacio (iD https://orcid.org/0000-0001-6034-695X, Carlos Ortiz-Hernández, Dylan Acosta- \\ Toscano, Robert Begambre-Meza, Blass Troncoso-Mendoza \\ Universidad de la Costa CUC, Barranquilla, Colombia

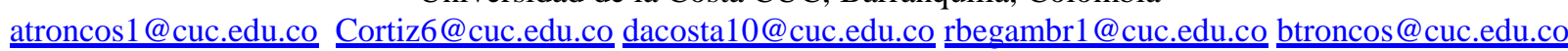

\begin{abstract}
Resumen
En este documento se demuestran los beneficios de la aplicación de las herramientas básicas de calidad en los procesos de extrusión de plásticos. Donde se realizó un estudio del proceso de estiramiento del plástico mediante el diagrama de Ishikawa y la implementación de rodillos de tracción. Además, se encontraron las causas de los defectos presentados en los rodillos de plástico; por lo tanto, una vez que se analizaron los resultados, se sugirió que se implementaran medidas de control en los procesos de inspección de la materia prima para determinar si se lograría controlar adecuadamente. También en el transporte de ella, debido a que los operadores no tenían en cuenta el debido proceso de carga y descarga de los rollos de plástico. Se analizó también los cronogramas de producción, donde se hallaban las mayores concentraciones de defectos mediante el uso de gráficos de control, para descartar el factor humano como una de las causas más relevantes de defectos en el proceso de producción. También en el almacenamiento de los rollos para evitar la formación de daños que causen las no conformidades del proceso del tejido de las bolsas de plástico.
\end{abstract}

\section{Palabras clave}

Tratamiento de Plásticos, Herramientas de calidad, Extrusión de Plástico, Gráfico de Pareto, Gráfico de Control, Diagrama de Ishikawa.

\begin{abstract}
This document demonstrates the benefits of the application of basic quality tools in plastic extrusion processes. Where a study of the plastic stretching process was carried out using the Ishikawa diagram and the implementation of traction rollers. In addition, the causes of the defects presented in the plastic rollers were found; therefore, once the results were analyzed, it was suggested that control measures be implemented in the inspection processes of the raw material to determine if adequate control could be achieved. Also in the transport of it, because the operators do not take into account the due process of loading and unloading of the plastic rolls. The production schedules were also analyzed, where there were the highest concentrations of defects through the use of control charts, to rule out the human factor as one of the most relevant causes of defects in the production process. Also in the storage of the rolls to avoid the formation of damages that cause the nonconformities of the process of the fabric of the plastic bags.
\end{abstract}

\section{Keywords:}

Plastics Treatment, Quality Tools, Plastic Extrusion, Pareto Chart, Control Chart, Ishikawa Diagram 
BILO Vol. 1 no. 1, Julio - Diciembre de 2019

\section{INTRODUCCIÓN}

La aplicación del control de la calidad en las distintas fases de los procesos productivos son más que necesarias para mejorar el resultado final de la actividad. Las grandes empresas y corporaciones invierten grandes cantidades de tiempo y recursos en el aseguramiento de la calidad de sus productos, esto se ve reflejado en la reputación que se logra forjar ya que su marca termina siendo asociada a productos de alta calidad que logran no solo satisfacer sino también superar las expectativas originales del cliente. A pesar de las bondades que ofrece la aplicación de procesos de calidad en la producción, este tipo de procesos siempre son costosos y esta es una de las principales razones por las cuales empresas que por la cantidad de producción, inventario, maquinaria, empleados y en general pasivos y activos que poseen se consideran de tamaño pequeño y mediano no aplican o poseen un plan de calidad o si utilizan procesos de calidad es solamente para cumplir con los requisitos mínimos que requieren su producción porque consideran que se deben reducir al mínimo los procesos de control de calidad con el fin de recortar gastos, esto desde el punto de vista de la producción podría parecer acertado ya que a mayor producción mayores ganancias porque existe mayor cantidad de producto en inventario que se traduce en ingresos, pero esta idea no solo no es acertada sino que también puede ser contraproducente, un proceso de calidad mínimo permite un aumento de la variabilidad en la producción que resulta en problemas de tipo directo y/o indirecto. Mediante la implementación de herramientas de calidad en este caso específico se implementarán el uso del diagrama de Ishikawa en conjunto con el diagrama de Pareto.

\section{ESTUDIOS PRIMARIOS DE LA LITERATURA}

En este artículo se habla sobre los sistemas de gestión de calidad y análisis de puntos de control crítico para las industrias alimentarias, la aplicación de estos sistemas de gestión en conjunto con los diagramas de Pareto e Ishikawa facilita la trazabilidad del producto y evaluación de riesgos. [1], además [2] aplico el uso de los diagramas de Pareto e Ishikawa en una refinería ubicada en Polonia, analizando las barras de acero obtenidas del proceso de laminado con el fin de recopilar información y hallar las causas más comunes de la no conformidad en el producto terminado, buscando la disminución del número de producto rechazado por ser no conforme. El diagrama de Ishikawa se utiliza con el fin de recolectar información sobre los causales de la no conformidad en el producto y el diagrama de Pareto se utilizó para segregar los resultados de tal manera que se lograra identificar la causa a la que se le atribuye la mayor cantidad de disconformidades en las barras de metal. (Sakao, T. Sundin, E) En este estudio se utiliza el diagrama de Ishikawa o espina de pescado basado en el análisis de los procesos de re manufactura desde un punto de vista teórico que se planea a llevar a cabo mediante un análisis de causa - efecto y un análisis de medios y fines, para esto el diagrama de Ishikawa es diseñado de tal manera que no solo se tengan en cuenta las $5 \mathrm{M}$ del modelo original sino agregar otros dos tópicos que serían comercialización y mantenimiento con el fin de agregar valor al cliente. [3], también [4] utilizaron como herramienta de calidad para mejorar los tiempos de búsqueda de la información del sistema de gestión de una compañía de servicios de salud el software libre Eclipse Process Framework Composer, por otro lado, (Moreira, L.M., Loos, M.J. 2018) Dicen que, a través del diagrama de Ishikawa, fue posible observar otros problemas como la ausencia de controles de producción y pérdidas, el monitoreo no directo de los empleados involucrados en las operaciones y los desgloses en el stock de materias primas. La producción entonces necesita ajustarse para satisfacer las demandas de ventas. [5], (de Souza, R.M., de Francisco, A.C., Braga, A.C., Coelho, A.M.S.) en su investigación apuntan que el diagrama de Ishikawa se considera una de las siete herramientas de calidad más utilizadas en el proceso de la mayoría de las organizaciones al presentar inmensas oportunidades para aprovechar la calidad y prevenir fallas en el proceso. Este artículo ha demostrado, sin pretender agotar el enfoque sobre el tema, que la gestión de la calidad se caracteriza por ser extremadamente importante para las organizaciones actuales. [6]. Por otro lado, [7] utilizaron la Simulación de Eventos discretos para mejorar los tiempos de respuesta de un laboratorio de control de la calidad en el sector farmacéutico. También (Restrepo Tamayo, L.M.) Afirma en su artículo que el uso de los cuadros de control, para controlar el espesor de la pintura de lámina de aluminio, permite reducir el reprocesamiento por aplicación y optimizar el consumo de materia prima. De esta manera, es evidente que las estadísticas industriales siguen siendo válidas en nuestras organizaciones. [8], (R Cahyana 2018 IOP Conf. Ser.: Mater. Sci. Eng. 434 012050) La implementación del diagrama de Ishikawa no se limita solamente a procesos industriales también puede usarse en la recopilación de información preliminar para el desarrollo de sistemas de información y en conjunto con su aplicación en procesos de control estadístico se puede asignar un porcentaje de responsabilidad a cada causa relacionada a un problema en determinada categoría produciendo soluciones alternativas. [9], Zasadzień, M., Midor, K. Statistical Process Control as a Failure Removal Improvement Tool, (2018), en este artículo se habla sobre la implementación de los sistemas de control continuo aplicando las herramientas de calidad con el fin de 
obtener una reducción en los tiempos muertos de los procesos obteniendo una mejora en la eficiencia y eficacia. [10], Luca Liliana 2016 IOP Conf. Ser.: Mater. Sci. Eng. 161 012099, En este articulo la autora no solo plantea el uso del diagrama de Ishikawa sino que incluso propone un nuevo modelo donde existen tres grandes subdivisiones causales del problema de las cuales se derivan unas subcausas siendo estas las $6 \mathrm{M}$ del diagrama de Ishikawa original. [11], cabe anotar que existen muchas metodologías de calidad como por ejemplo, la metodología de Diseño de Experimento Taguchi, utilizada por [12], donde los investigadores encontraron que la Densidad de Relleno y la Velocidad de Impresión son los parámetros más influyentes en el tiempo de impresión en el Modelado por Deposición Fundida 3D.

\section{METODOLOGÍA}

Para la resolución de la actividad investigativa se ubicó una empresa y se definió el proceso a estudiar en la actividad operativa, para esto se realizó una investigación de la función productiva de la empresa y una descripción del proceso que se va analizar, lo siguiente es realizar las actividades de recopilación de datos respectivas para poder utilizar las herramientas básicas de calidad, con el fin de obtener una serie de medidas o indicaciones que incidan de manera positiva en el proceso y demostrar la efectividad de su aplicación

\section{DESARROLLO}

Se realiza la recopilación de la información mediante la aplicación de un diagrama de Ishikawa de tal manera que se puedan clasificar las distintas causas potenciales de los rollos defectuosos en el proceso de laminado

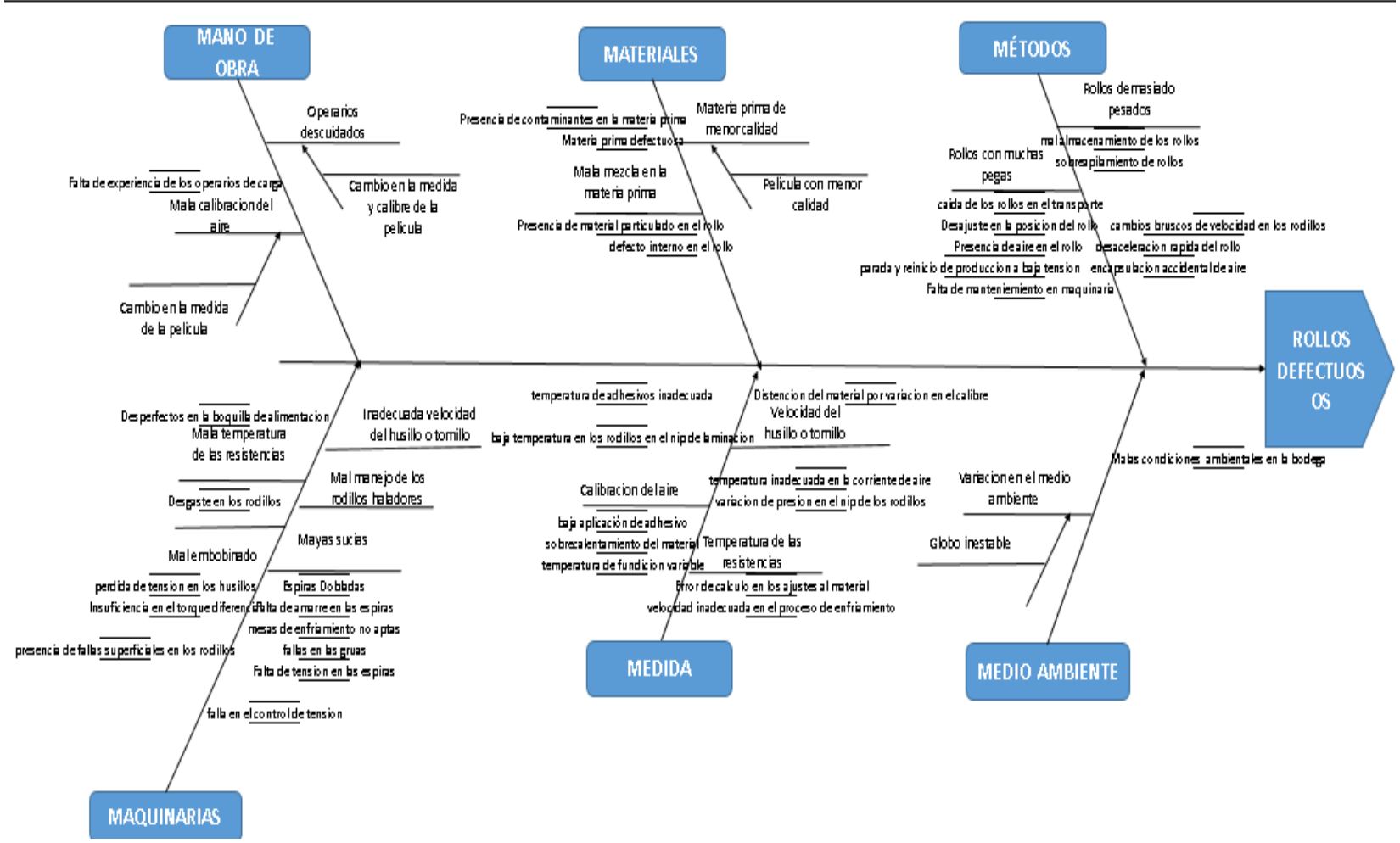

Fig. 1 Diagrama de Ishikawa fuentes: Autores 
BILO Vol. 1 no. 1, Julio - Diciembre de 2019

Se tabulan los datos en una tabla y se procede a asignarles un identificador para su representación gráfica.

\begin{tabular}{|c|c|c|c|c|c|c|c|}
\hline $\begin{array}{c}\text { Datos } \\
\text { recolectados }\end{array}$ & $\begin{array}{l}\text { ID en } \\
\text { gráfico }\end{array}$ & \multicolumn{3}{|c|}{ Posición real (Causas y datos ordenados) } & $\begin{array}{l}\text { Frecuencia } \\
\text { acumulada }\end{array}$ & Porcentaje & $\begin{array}{l}\text { Porcentaje } \\
\text { acumulado }\end{array}$ \\
\hline 3 & P1 & 1 & rollos defectuosos & 30 & 30 & $14 \%$ & $14 \%$ \\
\hline 2 & $\mathrm{P2}$ & 2 & mala medida de la pelicula & 8 & 38 & $4 \%$ & $4 \%$ \\
\hline 6 & P3 & 3 & rollos con muchas pegas & 8 & 46 & $4 \%$ & $4 \%$ \\
\hline 4 & P4 & 4 & parada y reinicio de produccion a baja tension & 8 & 54 & $4 \%$ & $4 \%$ \\
\hline 1 & P5 & 5 & baja aplicación de adhesivo & 7 & 61 & $3 \%$ & $3 \%$ \\
\hline 8 & P6 & 6 & mala mezcla de la materia prima & 6 & 67 & $3 \%$ & $3 \%$ \\
\hline 5 & P7 & 7 & Malas condiciones ambientales en la bodega & 6 & 73 & $3 \%$ & $3 \%$ \\
\hline 2 & P8 & 8 & Presencia de aire en el rollo & 6 & 79 & $3 \%$ & $3 \%$ \\
\hline 8 & $\mathrm{Pg}$ & 9 & falla en el control de tension & 6 & 85 & $3 \%$ & $3 \%$ \\
\hline 4 & $\mathrm{P} 10$ & 10 & cambios bruscos de velocidad en los rodillos & 6 & 91 & $3 \%$ & $3 \%$ \\
\hline 1 & P11 & 11 & mal embobinado & 5 & 96 & $2 \%$ & $2 \%$ \\
\hline 5 & P12 & 12 & operarios descuidados & 5 & 101 & $2 \%$ & $2 \%$ \\
\hline 30 & $\mathrm{P} 13$ & 13 & Falta de amarre en las espiras & 5 & 106 & $2 \%$ & $2 \%$ \\
\hline 3 & P14 & 14 & sobreapilamiento de rollos & 5 & 111 & $2 \%$ & $2 \%$ \\
\hline 5 & P15 & 15 & Falta de manteniemiento en maquinaria & 5 & 116 & $2 \%$ & $2 \%$ \\
\hline 4 & P16 & 16 & desaceleracion rapida del rollo & 5 & 121 & $2 \%$ & $2 \%$ \\
\hline 3 & P17 & 17 & encapsulacion accidental de aire & 5 & 126 & $2 \%$ & $2 \%$ \\
\hline 5 & P18 & 18 & mayas sucias & 4 & 130 & $2 \%$ & $2 \%$ \\
\hline 6 & P19 & 19 & rollos demasiado pesados & 4 & 134 & $2 \%$ & $2 \%$ \\
\hline 3 & $\mathrm{P} 20$ & 20 & mesas de enfriamiento no aptas & 4 & 138 & $2 \%$ & $2 \%$ \\
\hline 1 & P21 & 21 & Desgaste en los rodillos & 4 & 142 & $2 \%$ & $2 \%$ \\
\hline 3 & P22 & 22 & temperatura de adhesivos inadecuada & 4 & 146 & $2 \%$ & $2 \%$ \\
\hline 1 & P23 & 23 & presencia de fallas superficiales en los rodillos & 4 & 150 & $2 \%$ & $2 \%$ \\
\hline 4 & P24 & 24 & mala calibracion del aire & 3 & 153 & $1 \%$ & $1 \%$ \\
\hline 3 & P25 & 25 & Espiras Dobladas & 3 & 156 & $1 \%$ & $1 \%$ \\
\hline 2 & P26 & 26 & mal almacenamiento de los rollos & 3 & 159 & $1 \%$ & $1 \%$ \\
\hline 3 & P27 & 27 & caida de los rollos en el transporte & 3 & 162 & $1 \%$ & $1 \%$ \\
\hline 6 & P28 & 28 & Falta de experiencia de los operarios de carga & 3 & 165 & $1 \%$ & $1 \%$ \\
\hline 4 & P29 & 29 & Desajuste en la posicion del rollo & 3 & 168 & $1 \%$ & $1 \%$ \\
\hline 2 & P30 & 30 & Insuficiencia en el torque diferencial & 3 & 171 & $1 \%$ & $1 \%$ \\
\hline 7 & P31 & 31 & Error de calculo en los ajustes al material & 3 & 174 & $1 \%$ & $1 \%$ \\
\hline 2 & P32 & 32 & Presencia de material particulado en el rollo & 3 & 177 & $1 \%$ & $1 \%$ \\
\hline 3 & P33 & 33 & sobrecalentamiento del material & 3 & 180 & $1 \%$ & $1 \%$ \\
\hline 5 & P34 & 34 & variacion de presion en el nip de los rodillos & 3 & 183 & $1 \%$ & $1 \%$ \\
\hline 2 & P35 & 35 & defecto interno en el rollo & 3 & 186 & $1 \%$ & $1 \%$ \\
\hline 1 & P36 & 36 & temperatura de fundicion variable & 3 & 189 & $1 \%$ & $1 \%$ \\
\hline 3 & P37 & 37 & inadecuada velocidad del husillo o tornillo & 2 & 191 & $1 \%$ & $1 \%$ \\
\hline 2 & P38 & 38 & mal manejo en los gatos de los rodillos haladores & 2 & 193 & $1 \%$ & $1 \%$ \\
\hline 3 & P39 & 39 & perdida de tension en los husillos & 2 & 195 & $1 \%$ & $1 \%$ \\
\hline 2 & P40 & 40 & baja temperatura en los rodillos en el nip de laminacion & 2 & 197 & $1 \%$ & $1 \%$ \\
\hline 2 & P41 & 41 & temperatura inadecuada en la corriente de aire & 2 & 199 & $1 \%$ & $1 \%$ \\
\hline 3 & P42 & 42 & Presencia de contaminantes en la materia prima & 2 & 201 & $1 \%$ & $1 \%$ \\
\hline 4 & P43 & 43 & Materia prima defectuosa & 2 & 203 & $1 \%$ & $1 \%$ \\
\hline 3 & P44 & 44 & Desperfectos en la boquilla de alimentacion & 2 & 205 & $1 \%$ & $1 \%$ \\
\hline 8 & P45 & 45 & Distencion del material por variacion en el calibre & 2 & 207 & $1 \%$ & $1 \%$ \\
\hline 6 & P46 & 46 & mala temperatura de las resistencias & 1 & 208 & $0 \%$ & $0 \%$ \\
\hline 6 & P47 & 47 & mucha variacion en el medio ambiente & 1 & 209 & $0 \%$ & $0 \%$ \\
\hline 5 & P48 & 48 & fallas en las gruas & 1 & 210 & $0 \%$ & $0 \%$ \\
\hline 5 & P49 & 49 & Falta de tension en las espiras & 1 & 211 & $0 \%$ & $0 \%$ \\
\hline 3 & P50 & 50 & velocidad inadecuada en el proceso de enfriamiento & 1 & 212 & $0 \%$ & $0 \%$ \\
\hline
\end{tabular}

Las causas potenciales recopiladas en el diagrama de Ishikawa son monitoreadas con el fin de determinar la frecuencia con la que ocurren además de asignarles un identificador y una ponderación con el fin de realizar el diagrama de Pareto. 


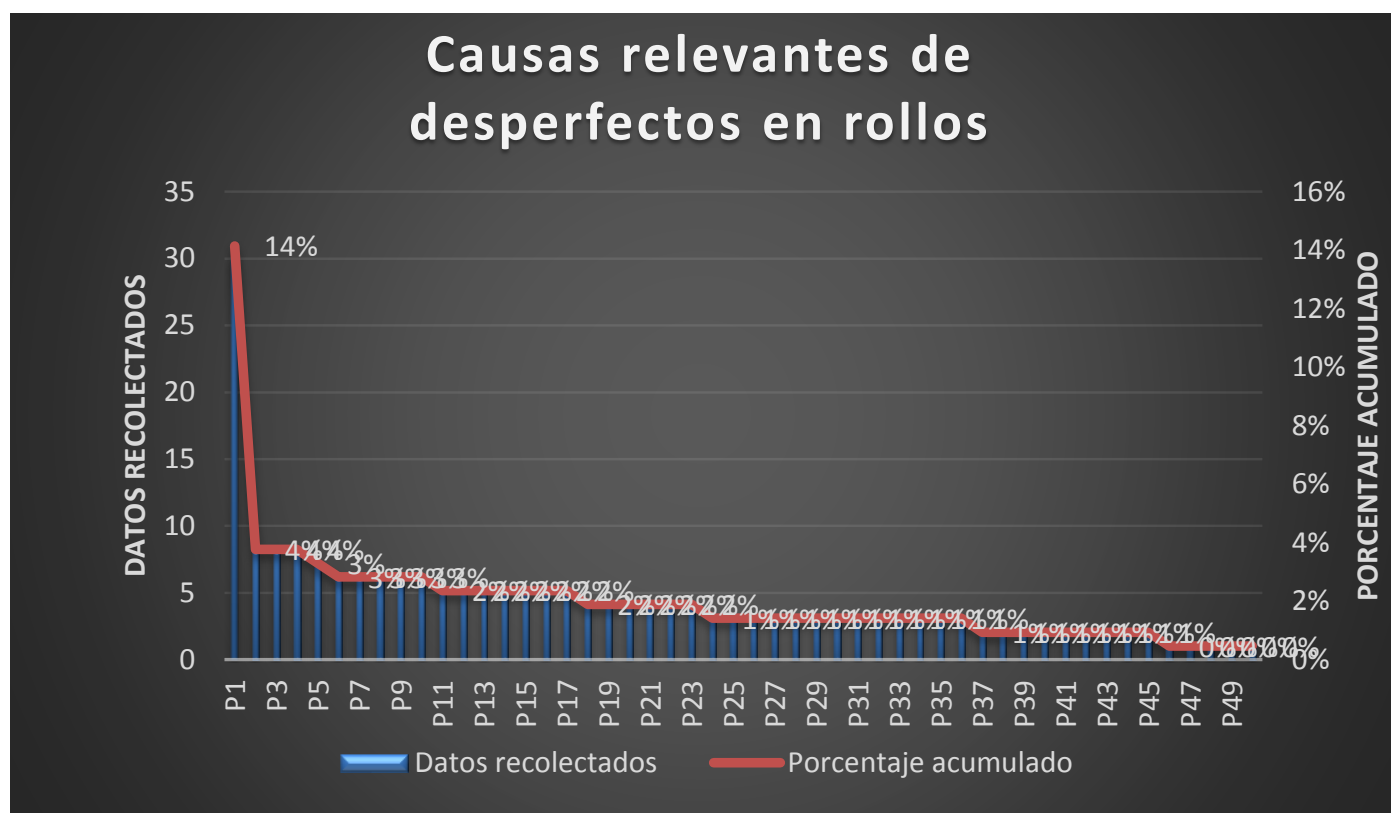

Fig. 2 Causas de desperfectos fuentes: Autores

De acuerdo con lo arrojado por el diagrama de Pareto las causas importantes a tener en cuenta en el proceso son aquellas más frecuentes como los rollos para laminado que presentan defectos previos. Luego de que se elabora los rollos en la maquina extrusora, estos pasan por las maquinas selladoras para así elaborar la bolsa de manigueta. Estas bolsas deben de tener una medida de ancho $25 \mathrm{~cm}$ y largo de $50 \mathrm{~cm}$. Para elaborar la carta XS se tomaron las medidas del ancho de la bolsa, se tomaron 14 muestras cada hora, esto hasta terminar un turno de trabajo. Los datos están en la siguiente tabla:

\begin{tabular}{|c|c|c|c|c|c|c|c|c|c|c|c|c|c|c|}
\hline \begin{tabular}{|l} 
hora/muestras $\mathrm{V}$ \\
\end{tabular} & 72 & 73 & 74 & $\nabla$ & 76 & 77 & $\nabla$ & 79 & $\nabla 10$ & $\nabla$ & $\nabla 1$ & $\nabla 13$ & 7 & $\nabla$ \\
\hline 6:00am & 25,8 & 25,3 & 24,4 & 26,4 & 24,5 & 26,6 & 26,1 & 25,1 & 26,4 & 24,1 & 26,1 & 24,5 & 27 & 25,8 \\
\hline 7:00am & 26,9 & 24,3 & 25,4 & 26,7 & 26,8 & 25,8 & 25,9 & 25,4 & 24,9 & 24,2 & 26,9 & 26,3 & 24,2 & 25,2 \\
\hline 8:00am & 26,6 & 24 & 25,9 & 26,6 & 26,9 & 26,7 & 26,4 & 24,6 & 24 & 25,4 & 25,4 & 25,3 & 24,3 & 26,3 \\
\hline 9:00am & 24,7 & 25,8 & 26,7 & 26,4 & 27 & 27 & 24,8 & 25,1 & 25,7 & 26,6 & 24,6 & 24,3 & 26,7 & 26,6 \\
\hline 10:00am & 25,5 & 25,5 & 24,2 & 26,2 & 27 & 25,5 & 24,6 & 26,6 & 26 & 26,6 & 25,7 & 24,6 & 26,7 & 24,1 \\
\hline 11:00am & 25,4 & 24,1 & 26,8 & 25,2 & 26,2 & 25 & 26,3 & 24 & 25 & 26,6 & 25,7 & 26,7 & 24,7 & 24,6 \\
\hline 12:00am & 24,5 & 26,1 & 26,9 & 24,6 & 25,7 & 26,6 & 24,1 & 25,5 & 26,5 & 24,8 & 24,6 & 24,8 & 26,2 & 25,2 \\
\hline 1:00am & 25,6 & 26,2 & 24,5 & 26,2 & 27 & 26,7 & 24,4 & 26 & 25,5 & 25,2 & 24,1 & 24,9 & 24,5 & 25,7 \\
\hline 2:00am & 26,1 & 24,3 & 25,7 & 24,6 & 26 & 24,4 & 24,5 & 24,3 & 26,7 & 27 & 26,2 & 25,9 & 25,4 & 26,5 \\
\hline 3:00am & 25,3 & 24,5 & 25,2 & 25,1 & 25,8 & 25,7 & 26,6 & 26,7 & 26 & 26 & 26,6 & 25,9 & 24,9 & 24,6 \\
\hline 4:00am & 26,6 & 25,9 & 24,9 & 26,5 & 24,9 & 24,2 & 26,9 & 25,4 & 24. & 25,2 & 26 & 26,9 & 24,9 & 25,1 \\
\hline 5:00am & 26,9 & 26,9 & 24,5 & 24 & 25,4 & 24 & 25,3 & 27] & 24,5 & 25,8 & 24,1 & 25 & 24 & 24,6 \\
\hline
\end{tabular}

Después de haber recopilado los datos se hallan las variables respecto a la carta de control. Ya con estos rangos de datos podemos construir los gráficos de control. Que nos queda así: Se procede a realizar la respectiva representación gráfica de los datos. 
BILO Vol. 1 no. 1, Julio - Diciembre de 2019

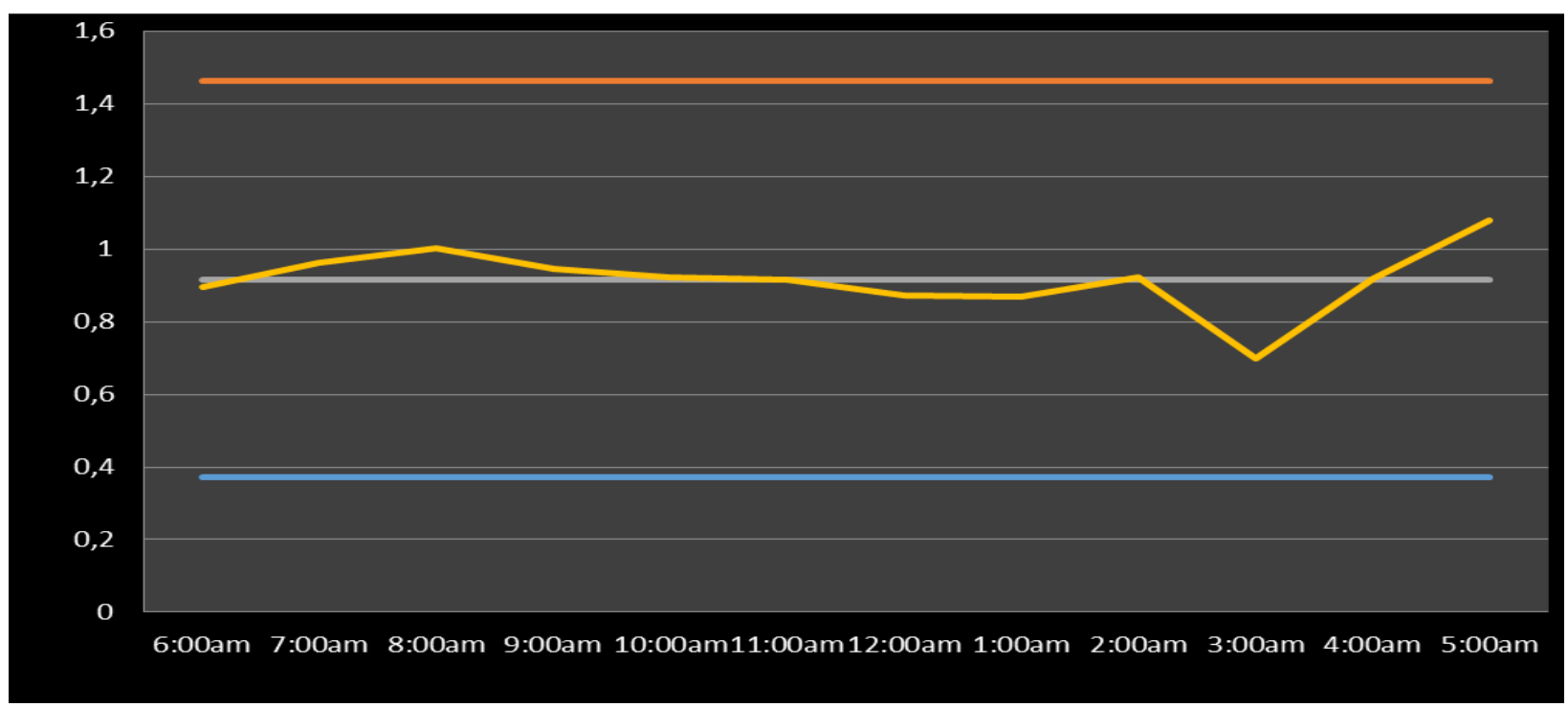

Fig. 3 Carta de control Fuente: Autores

\section{CONCLUSIONES}

El finalizar este análisis, se llega a la conclusión de que la aplicación de las herramientas de calidad es una parte fundamental que facilita enormemente el trabajo de control en los procesos mediante la resolución de problemas por medio de la identificación de sus causas. La utilidad práctica de los diagramas de causa-efecto se encuentra en la necesidad de analizar el un problema desde su origen, en producción no basta con tratar el problema sino que también debe evitar que se produzca otra vez, para esto se hace necesario examinar las causas de este por tanto el diagrama de causa-efecto o Ishikawa es útil porque brinda una clasificación en varios tópicos que engloban la mayoría de los causales posibles que pueden generar un problema lo que facilita la identificación de sus causas y posibles soluciones.

Según las herramientas de calidad aplicadas se le debe dar prioridad a aquellos pun-tos de mayor importancia como la materia prima defectuosa y mala operación de las máquinas de trabajo por parte de los empleados. Basándose en los datos recolectados debe hacerse una capacitación al personal, junto con incentivos para el buen desarrollo de las prácticas de trabajo debido a que la mayoría de las causas generadoras del problema se debe por malas prácticas en el desarrollo de las actividades de los trabajadores, ya sea por falta de experiencia o mala disposición en el desempeño de su labor.

\section{REFERENCIAS}

[1] B. Caballero, F. Finglas y F. Toldra, «Risk Assessment in Conjunction with Other Food Safety Tools Such as FMEA, Ishikawa Diagrams and Pareto,» Elsevier, 2016, p. 300.

[2] S. T. Dziuba, M. Ingaldi y M. Kadlubek, «Quality analysis of the steel bars in chosen metallurgical enter-prise,» de Tanger LTDA, Czech Republic, 2018.

[3] T. Sakao y E. Sundin, «How to Improve Remanufacturing?-A Systematic Analysis of Practices and Theories,» Journal of Manufacturing Science and Engineering, vol. 141, n 2, p. 13, 2018. 
[4] J. De-La-Hoz-Hernandez, A. Troncoso-Palacio y E. De-La-Hoz-Franco, «Implementation of the Eclipse Process Framework Composer Tool for the Documentation of Quality Management Systems: A Case Applied in Healthcare Services,» Lecture Notes in Computer Science, vol. 11656, pp. 200-210, 2019.

[5] L. Moreira, «Analysis of product supply breaks in a bakery through the Ishikawa diagram,» Espacios, vol. 39, $n^{\text {o } 3, ~ p . ~ 9, ~} 2018$.

[6] R. Souza, F. A y A. Coelho, «Resolution of the high level noise at area animal slaughtering and manufacturing: Application of Ishikawa diagram for improving the work environment,» Reseachgate, 2014.

[7] A. Troncoso-Palacio, D. Neira-Rodado, M. Ortiz-Barrios, G. Jiménez-Delgado y H. Hernández-Palma, «Using Discrete-Event-Simulation for Improving Operational Efficiency in Laboratories: A Case Study in Pharmaceutical Industry,» Lecture Note Computer Science, vol. 10942, pp. 440-451, 2018.

[8] L. Restrepo, «Control charts to optimize the process of painting on aluminum sheets,» Espacios, vol. 39, $\mathrm{n}^{\circ} 22$, p. 34, 2018.

[9] R. Cahyana, «A preliminary investigation of information system using Ishikawa diagram and sectoral statistics,» ResearchGate, 2018.

[10] M. Zasadzien y K. Midor, «Statistical Process Control as a Failure Removal Improvement Tool,» Sciendo, vol. $21, \mathrm{n}^{\mathrm{o}} 3$, p. 5, 2018.

[11] L. Liliana, «A new model of Ishikawa diagram for quality assessment,» de IOP Conference Series: Materials Science and Engineering.

[12] A. Sánchez-Comas, A. Troncoso-Palacio, S. Troncoso-Mendoza y D. Neira-Rodado, «Application of Taguchi Experimental Design for identification of factors influence over 3D Printing Time with Fused Deposition Modeling,» International Journal of Management Science \& Operations Research, vol. 1, nº 1, pp. 43-48, 2016. 